\section{Der akute Hirnschlag}

\author{
Zerebrovaskuläre Arbeitsgruppe der Schweiz (ZAS) und \\ Schweizerische Herzstiftung (SHS) ${ }^{1}$
}

\begin{abstract}
Der akute Hirnschlag ist zu 80-85\% ischämisch und zu 15-20\% durch intrazerebrale und subarachnoidale Blutungen bedingt. Pathophysiologisch liegt dem ischämischen Hirninfarkt eine lokale Thrombose, eine arterio-arterielle oder eine kardiogene Embolie zugrunde. Zwei von drei Infarkten betreffen das Karotisstromgebiet und einer von drei das vertebrobasiläre Territorium. Motorische und sensible Halbseitensyndrome, homonyme Gesichtsfelddefekte und neuropsychologische Ausfälle charakterisieren hemisphärische Insulte im Karotisstromgebiet, links mit Aphasien und rechts mit Raumverarbeitungsstörungen. Vertebrobasiläre Störungen äussern sich in bilateralen motorischen und sensiblen Störungen, Ataxien, Gesichtsfelddefekten, Doppelbildern, Schluckstörungen oder gekreuzten Hirnnerven- und Extremitätensymptomen. Ungefähr je $20 \%$ der Hirninfarkte haben eine extrakranielle oder intrakranielle Makroangiopathie, Mikroangiopathie oder kardio-embolische Ursache und wenige Prozent der Schlaganfälle beruhen auf einer nichtarteriosklerotischen Gefässkrankheit, z.B. einer Dissektion oder Koagulopathie. Makroangiopathien und Embolien führen typischerweise zu Territorialinfarkten und Mikroangiopathien zu Lakunen. Zeitlich gesehen können sich zerebrale Ischämien in Form transitorischer ischämischer Attacken oder als permanente neurologische Ausfälle äussern.
\end{abstract}

\footnotetext{
1 Folgende Personen haben zur Erstellung der Fachartikel beigetragen:

M. Arnold, Bern; A. Barth, Bern; C. Bassetti, Bern; R. Baumgartner, Zürich; S. Beer, Valens; J. Bogousslavsky, Lausanne; A. Carota, Lausanne; A. Carruzzo, Lausanne; G. Devuyst, Lausanne; A. Dupont, Basel; S. Engelter, Basel; P. Erne, Luzern; F. Fluri, Basel; R. Guzman, Bern; S. Heffet, Genève; HJ. Hungerbühler, Aarau; HG. Imhof, Zürich; E. Keller, Zürich; H. Keller, Zürich; J. Kesselring, Valens; J. Le Floch-Rohr, Genève; K. Loevblad, Bern; Ph. Lyrer, Basel; F. Mahler, Bern; A. Marx, Lugano; H. Mattle, Bern (Redaktor dieses Artikels); B. Meier, Bern; R. Mordasini, Bern; M. Mosso, Zürich; F. Müller, Scherzingen; S. Osswald, Basel; M. Reichhart, Lausanne; L. Remonda, Bern; A. Rivoir, Bern; D. Rüfenacht, Genève; W. Rutishauser, Genève; R. Sztaijzl, Genève; L. Regli, Lausanne; J. Rem, Basel; G. Schroth, Bern; C. Städler, Lugano; P. Stierli, Aarau; M. Sturzenegger, Bern; B. Tettenborn, St. Gallen; C. Tosi, Lugano; B. Weder, St. Gallen; S. Windecker, Bern.
}

Korrespondenz:

Prof. Heinrich Mattle

Neurologische Klinik und Poliklinik

Inselspital

CH-3010 Bern

Tel. 0316322111

Fax 0316329679

E-mail: heinrich.mattle@insel.ch

\section{Lernziele}

1. Kenntnis einfacher pathophysiologischer Zusammenhänge

2. Neurologische Leitsymptome der hemisphärischen Ischämien erkennen

3. Leitsymptome der vertebrobasilären Zirkulationsstörungen erkennen

4. Kenntnis des Begriffs der TIA

5. Kenntnis der wichtigsten ätiologischen Gruppen der Hirninfarkte

\section{Pathophysiologie}

Der akute ischämische Hirnschlag ist Folge einer lokalen Minderdurchblutung. Damit stehen dem Stoffwechsel der Neurone und Gliazellen zu wenig Sauerstoff und Glucose zur Verfügung. Sinkt der Blutfluss unter die Funktionsschwelle in den Bereich von 8-22 ml/100 g Hirngewebe/Minute, so fallen die elektrischen neuronalen Funktionen aus und es resultiert ein klinisch erkennbares Defizit der betroffenen Hirnregion. Sinkt die Perfusion weiter und bleibt während Minuten bis Stunden tief, entstehen irreversible, strukturelle Schäden. Diese Schwelle nennt man Infarzierungsschwelle. Mit zunehmender Dauer der Hypoxie schwinden die energiereichen Substrate, ATP fällt ab, durch anaerobe Glykolyse entsteht Laktat und die Wasser- und Ionenhomöostase kann nicht mehr aufrechterhalten werden. Kalzium und Natrium fliessen nach intrazellulär, exzitatorische Aminosäuren wie Glutamat und freie Radikale werden freigesetzt und zerstören die Zellen. Am Anfang dieser Kaskade wird Wasser nach intrazellulär verschoben (zytotoxisches Hirnödem) und nach Untergang der Zellen sammelt sich Wasser extrazellulär an (vasogenes Hirnödem). Ein massives Hirnödem kann raumfordernd wirken und den intrakraniellen Druck erhöhen.

Die schwersten Schäden finden sich in der Kernzone einer fokalen Ischämie, wo in der Regel die Infarzierungsschwelle rasch und anhaltend unterschritten wird. In der Randzone genügt die Blutzufuhr über Kollateralen oft, um den Strukturstoffwechsel aufrechtzuerhalten. Dieser Bereich zwischen Funktionsund Infarzierungsschwelle heisst Penumbra (Abb. 1). In der Penumbra besteht eine instabile Hirnzirkulation. Hält die Mangeldurchblutung zu lange an, gehen die Neurone und Gliazellen zugrunde. Kommt es genügend rasch zu einer Verbesserung der Zirkulation, überleben die Hirnzellen und können ihre Funktion wieder aufnehmen. Damit eröffnet sich eine therapeutische Chance. Gelingt es, die Blutzufuhr durch Rekanalisation des verschlossenen Gefässes vor dem Tod aller Zellen wieder in Gang zu bringen, resultieren ein kleinerer Infarkt und klinisch eine geringere Behinderung. 


\section{Abbildung 1}

In der Kernzone einer fokalen Ischämie (schwarz schraffiert) fehlt die Blutzufuhr in der Regel völlig und die Hirnzellen werden schon innerhalb von wenigen Minuten irreversibel geschädigt. Zur Randzone eines ischämischen Gebietes fliesst eine Restmenge Blut über Kollateralen, zu wenig, dass die Neurone funktionieren können, aber genug, um ihre Struktur zu erhalten. Dieser Bereich mit einer instabilen Zirkulation zwischen Funktions- und Infarzierungsschwelle heisst Penumbra. Kommt es genügend rasch zu einer Verbesserung der Zirkulation, überleben die Hirnzellen und können ihre Funktion wieder aufnehmen (Modifiziert nach L. Symon, Acta Neurol Scand 1980; 62, suppl. 78, 175-190).

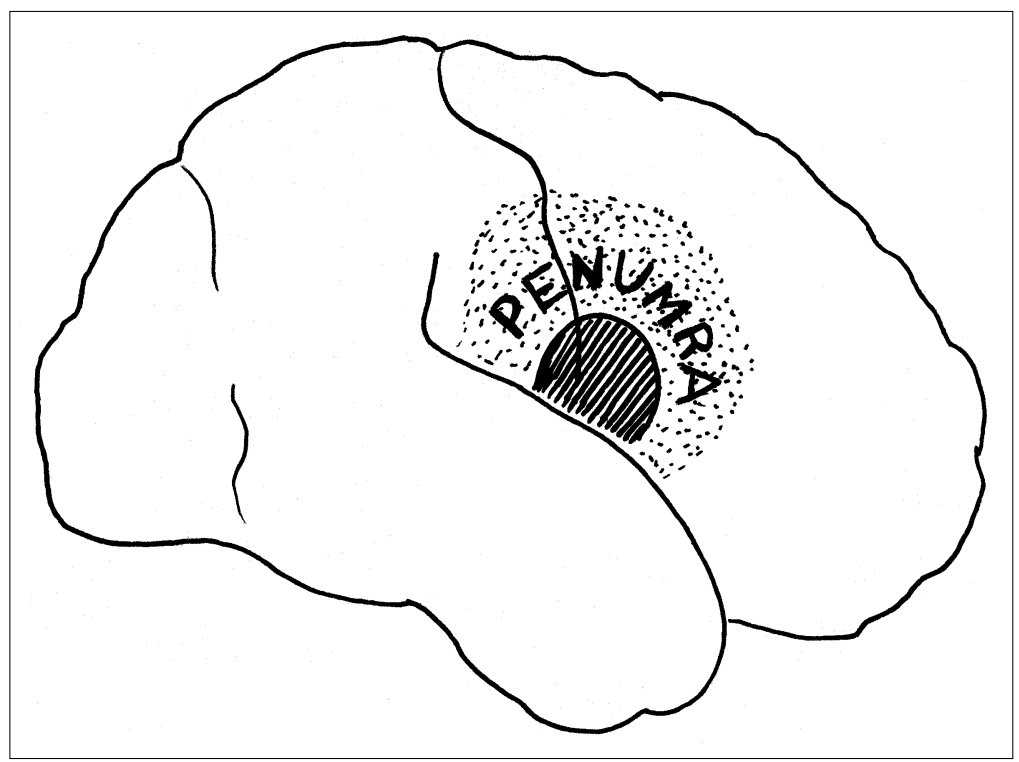

\section{Anatomische Bemerkungen}

Das Hirn wird über die Karotiden und die vertebrobasilären Arterien mit Blut versorgt. Die Arteria carotis interna (ICA) zweigt sich in die Arteria cerebri media (MCA) und Arteria cerebri anterior (ACA) auf. Sie stellt die Blutzufuhr zu den Frontal- und Parietallappen und anterolateralen Temporallappenanteilen sowie tiefen Strukturen der Hemisphären wie Basalganglien und Capsula interna sicher. Im weiteren versorgt die Arteria carotis interna (ICA) über die Arteria ophthalmica (OA) das Auge. Die Karotis und ihre Äste werden auch als vorderer Hirnkreislauf bezeichnet. Der hintere Hirnkreislauf wird aus den Vertebralarterien (VA) gespiesen, die sich zur Arteria basilaris (BA) vereinigen. Aus diesen Arterien entspringen 3 paarige Kleinhirnarterien und aus der Basilarisspitze entstammen die Arteriae cerebri posteriores (PCA) und die thalamoperforierenden Arteriolen. Somit versorgt das vertebrobasiläre System den Hirnstamm, das Kleinhirn, die Okzipitallappen, die inferomedialen Temporallappenanteile und die meisten Thalamuskerne. Bei jeder 7. bis 14. Person entspringen eine oder beide Arteriae cerebri posteriores aus der Karotis via Arteria communicans posterior. Bei vielen Verschlüssen und Stenosen stellt ein wirksames Kollateralsystem eine ausreichende Blutversorgung sicher. Extrakraniell können beispielsweise Ophthalmikaäste Blut von der Arteria carotis externa zur Arteria carotis interna oder über Muskeläste zur Arteria vertebralis bringen. Intrakraniell besteht über den
Circulus arteriosus Willisii an der Hirnbasis via die Arteria communicans anterior und die Arteriae communicantes posteriores ein besonders effizienter Umgehungskreislauf. Verschlüsse distal des Circulus arteriosus Willisii können teilweise über die leptomeningealen Gefässanastomosen an der Hirnoberfläche kompensiert werden.

\section{Klinische Symptome [1, 2]}

\section{Bewusstsein}

Eine verminderte Vigilanzlage (Schläfrigkeit, Sopor, Koma) weist bei unmittelbarem Auftreten zusammen mit anderen Symptomen auf einen Hirnstamminfarkt hin. Nimmt die Vigilanz im Laufe von Stunden bis wenigen Tagen ab, können ein grosser Hemisphäreninfarkt mit raumforderndem Ödem und Mittellinienverlagerung oder ein Kleinhirninfarkt mit Hirnstammkompression bestehen.

\section{Sprachstörungen}

Eine Aphasie weist auf einen linkshemisphärischen Infarkt hin. Bei Läsionen vor der Zentralregion ist der Sprachfluss in der Regel gestört (motorische oder nicht-flüssige Aphasie vom Typ Broca). Bei Läsionen hinter der Zentralregion bleibt der Sprachfluss erhalten (sensorische oder flüssige Aphasie vom Typ Wernicke). Mit dem Sprachverständnis verhält es sich umgekehrt. Bei der Broca Aphasie ist es erhalten, bei der Wernicke-Aphasie gestört. Eine Störung des Nachsprechens (Sprachrepetition; Leitungsaphasie) ist typisch für Läsionen im unteren Parietallappen oder im insulären Bereich (Läsion der Verbindung von "Wernicke zu Broca»). Eine Aphasie geht in der Regel mit einer Agraphie und Alexie einher. Im Gegensatz dazu sind bei der Dysarthrie Lesen und Schreiben ungestört und es werden keine "falschen Wörter" gesprochen. Die Sprache ist jedoch undeutlich und verwaschen bis hin zur Unverständlicheit. Dysarthrie hat eine nur geringe lokalisatorische Bedeutung. Hirnstammläsionen, aber auch Kleinhirn- oder rechtsund linkshemisphärische Läsionen können zur Dysarthrie führen. Mutismus, d.h. fehlende Sprache bei erhaltenem Bewusstsein, kann Teil einer akuten Aphasie sein, oder aber eine meist bilaterale mesencephale, diencephale oder frontoorbitale Läsion andeuten.

\section{Andere neuropsychologische Ausfälle}

Apraxien sind Störungen der Bewegungsausführung bei erhaltener Motorik. Sie können Bewegungen im Gesicht (fazio-bucco-linguale Apraxie) oder der Extremitäten (Extremitätenaproxie) betreffen. Sie sind meist mit einer Aphasie und damit linkshemisphärischen Läsionen assoziiert. Rein linksseitige Extremitätenapraxien können auch bei rechtsfrontalen Läsionen auftreten. Ein Neglekt bzw. eine Vernachlässigung einer Körperseite und Raumhälfte ist bei rechtshemisphärischen Läsionen häufiger und dauerhafter als bei linksseitigen. Geprüft wird ein Neglekt durch bilateral synchrone sensible Stimulation, wobei der 


\section{Abbildung 2}

Schematische Darstellung der häufigsten Ursachen der Hirninfarkte (Mumenthaler M, Mattle H. Neurologie. Thieme, Stuttgart, 1997).

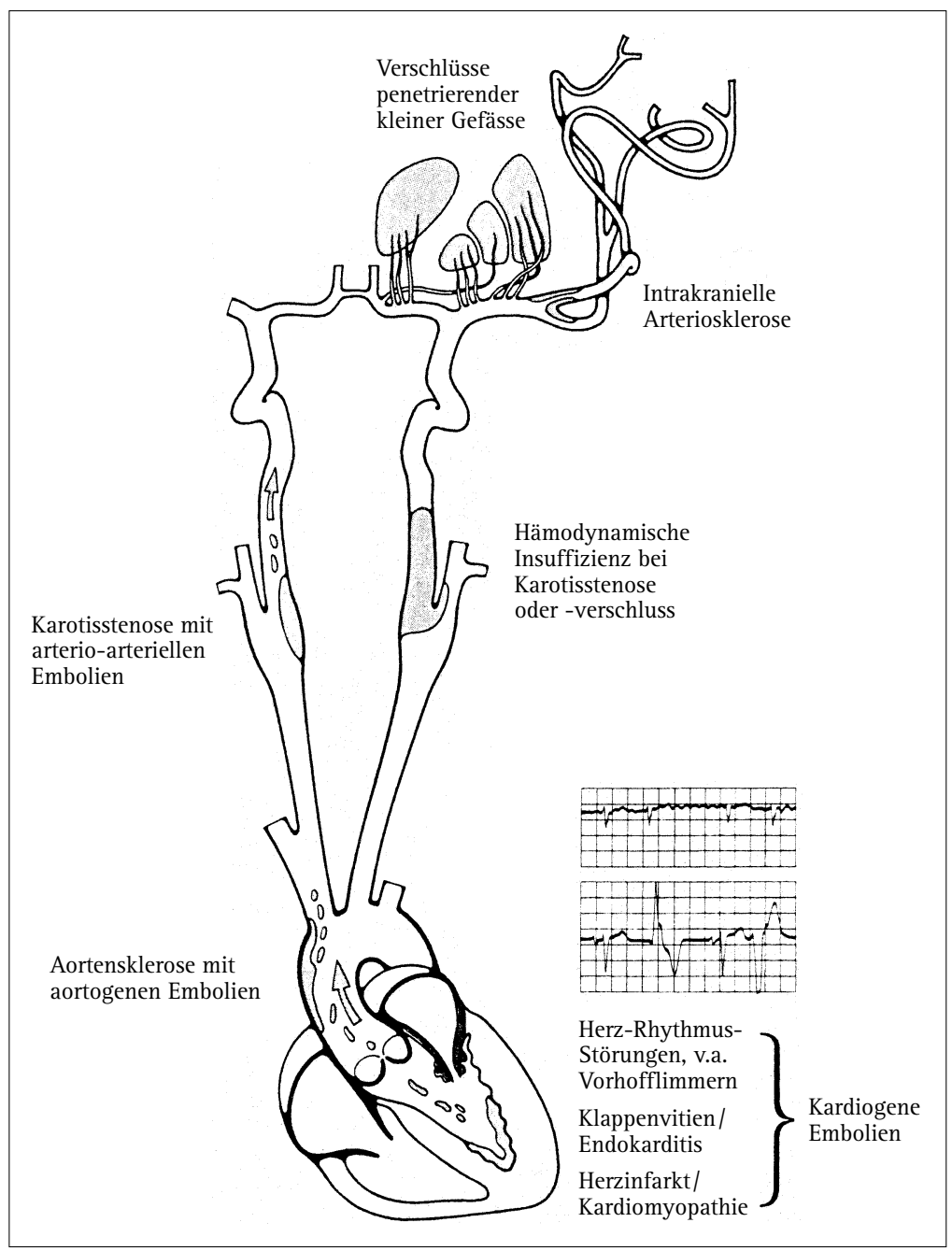

Patient mit Neglekt nur den Stimulus einer Seite wahrnimmt. Beim Zeichnen oder Kopieren eines Gegenstandes wird im Falle eines Neglekts das Zeichenblatt auf der Neglektseite leer bleiben oder nur die andere Hälfte gut gezeichnet. Eine Anosognosie bedeutet Nichterkennen des Krankseins und kommt bei grossen postzentralen, rechtshemisphärischen Läsionen vor. Raumsinnstörungen, erkennbar z. B. beim Zeichnen, sind häufig bei rechtshemisphärischen Läsionen, insbesondere des Parietallappens.

\section{Gesichtsfeldausfälle}

Gesichtsfeldausfälle können nur ein Auge betreffen (= monokulär) oder gleiche Gesichtsfeldausschnitte an beiden Augen (= homonyme Defekte). Monokuläre Ausfälle weisen in der Regel auf eine retinale Zirkulationsstörung hin, homonyme auf eine thalamische, temporale, parietale oder okzipitale. Parietal- und Okzipitallappenläsionen führen meist zu homonymen
Hemianopsien zur Gegenseite, Temporallappenläsionen zur gegenseitigen oberen Quadrantenanopsie. Altitudinale Hemianopsien kommen bei vertebrobasilären Zirkulationsstörungen im Okzipitallappen vor.

\section{Lähmungen und sensible Aufälle}

Lähmungen und sensible Aufälle treten meistens kombiniert auf. Sie weisen auf eine Läsion des gegenseitigen Kortex, der kortikobulbären oder kortikospinalen und aszendierenden sensiblen Bahnen hin. Bei kortikalen Läsionen ist oft und abhängig vom betroffenen Kortexareal die Motorik oder Sensibilität eines Körperteiles besonders schwer betroffen. Bei frontalen Läsionen steht die Parese, bei parietalen die Sensibilitätsstörung im Vordergrund. Läsionen der Bahnen, beispielsweise in der Capsula interna, verursachen ein homogen verteiltes Hemisyndrom von Gesicht, Arm und Bein auf der Gegenseite. Sind sensomotorische Ausfälle mit neuropsychologischen Störungen verbunden, liegt meist eine grosse Hemisphärenläsion vor. Treten Hemiparesen oder sensible Hemisyndrome isoliert und ohne weitere Begleitsymptome auf, so sind die zugrundeliegenden Läsionen klein. In der Regel handelt es sich um kleine, sogenannte lakunäre Infarkte subkortikal, in der Capsula interna oder im Hirnstamm ("pure motor stroke», "pure sensory stroke»).

\section{Störungen der Augenmotorik}

Eine "déviation conjuguée» zur Läsionsseite, die durch okulozephale Manöver überwindbar ist (= Gegenrollen der Augen bei passiver Kopfrotation), ist typisch für eine Hemisphärenläsion. Eine pontine Läsion führt hingegen zu einer horizontalen Blickparese, die durch okulozephale Manöver nicht überwindbar ist. Eine vertikale Blickparese ist meist mit einer Bewusstseinsstörung kombiniert und Ausdruck einer mesencephalen Schädigung. Eine internukleäre Ophthalmoplegie (INO), d.h. Lähmung des adduzierenden Auges und Nystagmus des abduzierenden Auges, weist auf eine einseitige tegmentale Läsion im Hirnstamm hin. Ein richtungsbestimmter bzw. Spontannystagmus ist typisch für eine Läsion im Hirnstamm, Kleinhirn oder auch der peripheren vestibulären Organe. Ein Blickrichtungsnystagmus weist auf eine zentrale Funktionsstörung im Hirnstamm oder Kleinhirn hin.

\section{Störungen der Pupillen}

Ungleich weite Pupillen können Ausdruck einer Okulomotoriusparese oder eines Hornersyndroms sein. Eine weite, auf Licht wenig oder nicht reagierende $\mathrm{Pu}$ pille kommt bei nukleären oder faszikulären Läsionen im Mittelhirn oder bei Läsionen im peripheren Verlauf des Nervus oculomotorius vor. Ein Hornersyndrom besteht aus einer engen, auf Licht reaktiven Pupille und einer Verengung der Lidspalte, am besten erkennbar bei Blick geradeaus oder nach unten. Es weist auf eine ipsilaterale Läsion der Sympathikusbahnen hin, entweder zentral im Hirnstamm oder peripher und dann meist perikarotideal wegen Karotisdissektion. 
Tabelle 1

Oberflächlicher (pialer) MCA-Infarkt [5].

\begin{tabular}{l}
\hline 1. Okklusion des vorderen, oberen Stammes \\
\hline sensomotorisches Hemisyndrom \\
\hline homonyme Hemianopsie \\
\hline globale oder Broca-Aphasie (linkshemisphärisch) \\
\hline Apraxie \\
\hline Hemineglekt (rechtshemisphärisch) \\
\hline 2. Okklusion des hinteren, unteren Stammes \\
\hline homonyme Hemi- oder Quadrantenanopsie \\
\hline Hemisyndrom, oft nur sensibel \\
\hline Wernicke-Aphasie (linkshemisphärisch) \\
\hline $\begin{array}{l}\text { Akalkulie, Fingeragnosie, Rechts-Links-Verwechslung und Agraphie } \\
\text { (= Gerstmann-Syndrom; linkshemisphärisch) }\end{array}$ \\
\hline Hemineglekt, Raumsinnstörung, Ankleide-Apraxie (rechtshemisphärisch) \\
\hline
\end{tabular}

\section{Tabelle 2}

Tiefer, striatocapsulärer MCA-Infarkt [6].

sensibles, motorisches oder sensomotorisches Hemisyndrom

Aphasie und Apraxie (linkshemisphärisch)

Hemineglekt (rechtshemisphärisch)

gelegentlich Bewegungsstörungen (Hemiballismus, Choreoathetose), akut oder mit Latenz auftretend

\section{Tabelle 3}

Kompletter MCA-Infarkt.

Kombination von $1 .+2$

Déviation conjugée zur Infarktseite

oft Schläfrigkeit

Anosognosie häufig (rechtshemisphärisch) sie mit ipsi- oder kontralateralen motorischen und sensiblen Ausfällen oder Ataxie kombiniert. "Gekreuzte Syndrome», d. h. einseitige nukleäre oder faszikuläre Hirnnervenläsionen kombiniert mit gegenseitigen motorischen oder sensiblen Hemisyndromen sind charakteristisch für Hirnstammläsionen. Einzig eine Karotisdissektion kann extraaxiale Infarkte oder Kompressionen der kaudalen Hirnnerven (IX-XII) verursachen und klinisch eine Hirnstammläsion vortäuschen [3].

\section{Zeitliche Präsentation}

Eine fokale zerebrale Ischämie führt innert Minuten zu Symptomen, die wieder innert Minuten verschwinden oder lebenslang bestehen bleiben können. Dauern die Symptome weniger als 24 Stunden, ist dies per definitionem eine transitorische ischämische Attacke (TIA), bleiben die Symptome länger als 24 Stunden bestehen, so handelt es sich um einen Infarkt. Eine TIA will nicht heissen, dass es sich nicht um einen Infarkt handelt. Im Gegenteil, kernspintomographisch finden sich in bis zu 80\% der TIAs fokale Signalstörungen als Ausdruck eines kleinen Infarktes. Zwecks differentialdiagnostischer Abgrenzung gegen Migräne, Tumor oder Abszess ist der akute Beginn mit initial maximaler Ausprägung der Ausfälle ein wichtiges Kennzeichen der Ischämie. Die volle Symptomatik muss sich aber nicht immer innert Minuten entwickeln. Sie kann auch progredient über Stunden auftreten und bei wiederholten neurologischen Untersuchungen stärker ausgeprägt sein (progressive stroke). Nicht selten, am häufigsten bei Karotisstenosen und am seltensten bei lakunären Läsionen, gehen einem Infarkt TIAs voraus. Betrifft eine Zirkulationsstörung die Retina, sprechen wir bei transitorischen Symptomen von Amaurosis fugax oder bei permanenten monokulären Ausfällen von einem Retinainfarkt.

\section{Klinische Syndrome}

Koordinationsstörungen, Extremitäten- und Gangataxie

Einer Extremitätenataxie liegt meistens eine Läsion der gleichseitigen Kleinhirnhemisphäre oder der Kleinhirnbahnen im Hirnstamm zugrunde. Ist der Kleinhirnwurm lädiert, betrifft die Ataxie vor allem den Körperstamm und kommt beim Stehen und Gehen besonders zum Vorschein. Eine Läsion der Hinterstränge oder peripheren Nerven äussert sich ebenfalls durch eine Stand- und Gangataxie, ist aber nur ausnahmsweise vaskulär bedingt. Eine sensible Störung wegen einem parietalen Infarkt kann zu einer kontralateralen Extremitätenataxie führen.

\section{Hirnnervenausfälle}

Hirnnervenausfälle weisen meist auf eine Hirnstammläsion hin. Isolierte nukleäre oder faszikuläre Läsionen sind selten und sind Ausdruck eines mesenzephalen (III, IV), pontinen (V bis VIII) oder medullären (V, IX bis XII) Infarktes. Weit häufiger sind
Abhängig vom Gefässterritorium, in dem sich eine Durchblutungsstörung ereignet, kommt es zu mehr oder weniger charakteristischen klinischen Syndromen.

Arteria cerebri media-Syndrom (Tabellen 1-3) Etwa die Hälfte der Hirninfarkte betreffen das Mediastromgebiet [4]. Ist der MCA-Hauptstamm verschlossen, so resultiert ein kompletter MCA-Infarkt oder bei guten leptomeningealen Kollateralen ein isolierter tiefer, subkortikaler MCA-Infarkt. Astverschlüsse führen zu oberflächlichen Infarkten, und die Okklusion penetrierender lentikulostriärer Äste hat meist Lakunen zur Folge.

Arteria cerebri anterior-Syndrome (Tabelle 4) Astverschlüsse sind häufiger als Hauptstammverschlüsse. Gelegentlich sind ACA-Insulte bilateral. 


\section{Tabelle 4}

Arteria cerebri anterior-Syndrome [7].

beinbetontes sensomotorisches Hemisyndrom

neuropsychologische Ausfälle wie Apathie, Abulie oder Mutismus, seltener Euphorie und Desinhibition, nichtflüssige Aphasie, Delirium

Greifreflex kontralateral, Harn-Stuhl-Inkontinenz

\section{Tabelle 5}

Arteria carotis interna-Syndrom [8].

transitorische oder permanente monokuläre Blindheit, selten lediglich Amaurosis fugax bei grellem Licht

TIA gelegentlich nur in Orthostase als «limb shaking TIA» [9]

hemisphärische Infarkte einzeln oder multipel, Symptome wie bei MCA- und ACA-Syndrom beschrieben

perikarotideales Syndrom (meist bei Karotisdissektion) mit Hals-Gesichts-Kopfschmerzen, Horner-Syndrom, ipsilateralen Hirnnervenausfällen und evtl. hemisphärischen Symptomen

\section{Tabelle 6}

Arteria cerebri posterior-Syndrom [10].

homonyme Gesichtsfeldausfälle, isoliert oder kombiniert mit

neuropsychologischen Ausfällen wie Amnesie, visuellen Wahrnehmungsstörungen,

Delirium

Alexie evtl. ohne Agraphie (linkshemisphärisch)

sensorische Aphasie (linkshemisphärisch)

Prosopagnosie (Nichterkennen vertrauter Gesichter; meist rechtshemisphärisch)

sensomotorisches Hemisyndrom, meist sensibel betont

kortikale Blindheit, oft mit Anosognosie (Anton-Syndrom; bilaterale Läsion)

\section{Tabelle 7}

Isolierte paramediane (vordere) Hirnstamminfarkte.

Thalamus: Hypersomnie, vertikale Blickparese, Amnesie [12, 13]

Mittelhirn (oft mit thalamischen Infarkten assoziert): Hypersomnie, faszikuläre oder nukleäre Okulomotoriusparese, vertikale Blickparese [14]

Pons: kontralaterales motorisches Hemisyndrom, Dysarthrie, oft nur flüchtige tegmentale Zeichen wie z.B. internukleäre Ophthalmoplegie, wenn bilateral Locked-inSyndrom [15]

Medulla: kontralaterales sensomotorisches Hemisyndrom, homolaterale HypoglossusParese

\section{Tabelle 8}

Isolierte (dorso-)laterale Hirnstamminfarkte.

Thalamus (Syndrom von Dejerine-Roussy): sensibles Hemisyndrom, Hemiataxie, sekundäres zentrales Schmerzsyndrom

Mittelhirn (selten): ipsilaterale Okulomotoriusparese, kontralaterales motorisches oder sensibles Hemisyndrom, Hemiataxie

Pons: ipsilaterale Hirnnervenausfälle (V-VII), horizontale ipsiversive Blickparese, kontralaterale motorische oder sensomotorische Ausfälle

Medulla (Syndrom von Wallenberg): gekreuzte und dissoziierte Sensibilitätsausfälle (homolateral in Gesichtshälfte, kontralateral am Hemikörper); ipsilaterales HornerSyndrom und IX-X-Parese mit Schluckstörungen und Heiserkeit; zentralvestibuläre Ausfälle (Vertigo, Nystagmus, Gangataxie), Atmungsstörungen
Arteria carotis interna-Syndrom (Tabelle 5) Symptome von seiten der Retina, sei dies eine Amaurosis fugax oder ein Retinainfarkt wegen Verschluss der Arteria centralis retinae, kombiniert mit ipsilateralen Hemisphärensymptomen beweisen ein Arteria carotis interna-Syndrom. Viel häufiger treten aber retinale und Hemisphärensymptome isoliert transitorisch oder permanent auf.

Arteria cerebri posterior-Syndrom (Tabelle 6) PCA-Insulte stellen etwa 10\% aller Hirninfarkte dar, treten meist einseitig auf und können bei Embolien auch bilateral sein. Oberflächliche Infarkte sind häufiger als komplette bei PCA-Hauptstammverschlüssen, bei denen auch der Thalamus infarziert. Dann kann der PCA-Infarkt einem kompletten MCA-Insult täuschend ähnlich sein.

Wenn die PCA aus der ICA entspringt (persistierende fötale Zirkulation) kann ein PCA-Infarkt mit einer Karotispathologie assoziiert sein. Normalerweise zeigen PCA-Infarkte jedoch eine vertebrobasiläre Zirkulationsstörung an.

Arteria basilaris- und Arteria vertebralisSyndrome (Tabellen 7-10)

Vertebrobasiläre Infarkte stellen 15-20\% aller Hirninfarkte dar. Isolierte paramediane und laterale Hirnstamminfarkte sowie kleine isolierte Kleinhirninfarkte treten meist wegen Mikroangiopathien auf [11]. Dorsale Hirnstamminfarkte sind nahezu immer mit Kleinhirninfarkten kombiniert. Isolierte grössere Kleinhirninfarkte sind häufig embolischer oder arteriosklerotischer Natur. Bei Thrombosen oder Embolien in Arteria vertebralis oder Arteria basilaris werden Hirnstamm und Kleinhirn in der Regel gleichzeitig betroffen.

\section{Lakunäre Syndrome (Tabelle 11)}

Sie machen etwa 20\% aller Infarkte aus und entstehen im Versorgungsgebiet penetrierender kleiner Arterien. Entsprechende klinische Syndrome sind in Tabelle 11 gelistet.

Lakunäre Syndrome können klinisch nicht mit Sicherheit dem karotidealen oder vertebrobasilären Stromgebiet zugeordnet werden. Hierzu ist eine Bildgebung erforderlich.

\section{Wasserscheidensyndrome}

Sie sind selten und treten typischerweise in Orthostase oder bei Hypotension auf. Grundlage sind hochgradige, hämodynamisch wirksame Stenosen oder Verschlüsse meist mehrerer Hirnarterien. Die verursachten Infarkte sind eventuell multipel [19]. "Limbshaking»-TIAs im Stehen sind charakteristisch. Ein Hemisyndrom ist in der Regel beinbetont.

\section{Ätiologie (Tabellen 12 und 13, Abbildung 2)}

Anamnestische Angaben wie vaskuläre Risikofaktoren oder Herzleiden und das klinische Syndrom ergeben oft einen verlässlichen Hinweis auf die Ätio- 


\section{Tabelle 9}

Kleinhirninfarkte [16].

"pseudovestibuläres» Syndrom: Drehschwindel, Gangstörung, Nystagmus

Kopfschmerzen und Somnolenz bei raumforderndem Ödem

zerebelläre Ausfälle: ipsi- oder bilaterale Extremitäten- und Rumpfataxie sowie

Fallneigung und Dysarthrie

evtl. dorsale Hirnstammzeichen

\section{Tabelle 10}

Kombinierte Hirnstamm-Kleinhirninfarkte.

Basilaristhrombose: bilaterale sensomotorische und Hirnnervenausfälle,

Kleinhirnzeichen, Augenbewegungsstörungen, Kopfschmerzen, Koma

Basilarisspitzensyndrom: Kombination von Zeichen von Infarkten im PCA- und thalamo-mesencephalen Bereich [17]

\section{Tabelle 11}

Lakunäre Syndrome [18].

\begin{tabular}{l}
\hline "pure motor stroke» \\
\hline "pure sensory stroke» \\
\hline "dysarthria clumsy hand" \\
\hline "ataxic hemiparesis" \\
\hline
\end{tabular}

\section{Tabelle 12}

Hauptgruppen ischämischer Hirninfarkte nach ätiologischen Gesichtspunkten.

\begin{tabular}{l}
\hline 1. Arteriosklerose grosser intra- und extrakranieller Hirngefässe \\
\hline a) Thrombose \\
\hline b) hämodynamische Insuffizienz \\
\hline c) arterio-arterielle Embolien \\
\hline 2. Kardiogene und aortogene Embolien \\
\hline 3. Erkrankung kleiner Hirngefässe / Lakunen \\
\hline 4. Nichtarteriosklerotische Ursachen \\
\hline a) Vaskulopathien \\
\hline b) Koagulopathien \\
\hline 5. Ätiologie unklar \\
\hline
\end{tabular}

logie eines Schlaganfalls. Damit kann die Abklärung entscheidend gesteuert und diagnostische «Rumdumschläge» können vermieden werden. Abbildung 2 und Tabelle 12 stellen die wichtigsten Ursachen von Hirninfarkten dar [20]. Tabelle 13 gibt eine ausführliche ätiologische Klassierung wieder [21]. Sie stellt eine Liste der Ätiologien von Schlaganfällen dar, die vor allem bei jüngeren Patienten zu suchen sind. Bei jüngeren Patienten sind arteriosklerotische Ursachen seltener als bei älteren. Einige nichtarteriosklerotische Krankheitsbilder werden in einem späteren Beitrag eingehend beschrieben. Für die Akuttherapie eines Hirninfarktes spielt die Ätiologie - von selte- nen Ausnahmen abgesehen - eine untergeordnete Rolle; sie ist jedoch für eine gezielte Sekundärprophylaxe entscheidend.

\section{Literatur}

1 Bassetti C, Bogousslavsky J. Klinische Symptome und Syndrome des Hirnschlags. Ther Umschau 1996;53:519-27.

2 Ginsberg M, Bogousslavsky J (eds). Cerebrovascular disease: pathophysiology, diagnosis, management. Boston: Blackwell Scientific Publications; 1998.

3 Sturzenegger M, Huber P. Cranial nerve palsies in spontaneous carotid artery dissection. J Neurol Neurosurg Psychiat 1993; 56:1191-9.

4 Bogousslavsky J, Van Melle G, Regli F. The Lausanne stroke registry. Analysis of 1000 consecutive patients with first stroke. Stroke 1988;19:1083-92.

5 Bogousslavsky J, Van Melle G, Regli F. Middle cerebral artery pial territory infarcts. A study of the Lausanne stroke registry. Ann Neurol 1989;4:555-60.

6 Donnan GA; Bladin PF, Berkovic SF, Longley WA, Saling MM. The stroke syndrome of striato-capsular infarction. Brain 1991;114:51-70.

7 Bogousslavsky J, Regli F. Anterior cerebral artery territory infarction in the Lausanne stroke registry. Arch Neurol 1990; 47:144-50

8 Bogousslavsky J, Regli F, Zografos L, Uske A. Optico-cerebral syndrome: simultaneous hemodynamic infarction of optic nerve and brain. Neurology 1987;37:263-8.

9 Baumgartner RW, Baumgartner I. Vasomotor reactivity is exhausted in transient ischaemic attacks and limb shaking. J Neurol Neurosurg Psychiatry 1998;65:561-4.

10 Pessin MS, Lathi ES, Cohen MB, Kwan ES, Hedges TR, Caplan LR. Clinical features and mechanisms of occipital infarction. Ann Neurol 1987;21:290-9.

11 Fisher CM, Caplan LR. Basilar artery branch occlusion. A cause of pontine infarction. Neurology 1971;21:900-5.

12 Bassetti C, Mathis J, Gugger M, Loevblad K, Hess CW. Hypersomnia following thalamic stroke. Ann Neurol 1996; 39:471-80.

13 Bogousslavsky J, Regli F, Uske A. Thalamic infarcts. Clinical syndromes, etiology and prognosis. Neurology 1988; 38:837-48.

14 Castaigne P, Lhermitte F, Buge A, Escourelle R, Hauw JJ, Lyon-Caen 0. Paramedian thalamic and midbrain infarcts. Clinical and neuropathological study. Ann Neurol 1981; 10:127-48.

15 Bassetti C, Bogousslavsky J, Barth A, Regli F. Isolated infarcts of the pons. Neurology 1996;46:165-74.

16 Amarenco P. The spectrum of cerebellar infarctions. Neurology 1991;41:973-9.

17 Caplan LR. «Top of the basilar» syndrome. Neurology 1980; 30:72-9.

18 Donnan GA, Norrving B, Bamford JM, Bogousslavsky J (eds). Lacunar and other subcortical infarctions. 0xford: 0xford University Press; 1995.

19 Bogousslavsky J, Regli F. Unilateral watershed cerebral infarcts. Neurology 1986;36:373-7.

20 Adams HP Jr, Bendixen BH, Kapelle LJ et al. Classification of subtype of acute ischemic stroke. Definitions for use in a multicenter clinical trial. TOAST. Trial of Org 10172 in Acute Ischemic Stroke Treatment. Stroke 1993;24:35-41.

21 Mumenthaler M, Mattle H. Neurologie. 10. Auflage Stuttgart: Thieme Verlag; 1997.

Sonderdrucke sind ab Herbst 2000 erhältlich bei der Schweizerischen Herzstiftung, Schwarztorstr. 14, Postfach, 3000 Bern 14.

\section{La version française suivra}




\section{Tabelle 13}

Ätiologische Klassierung ischämischer Hirninfarkte.

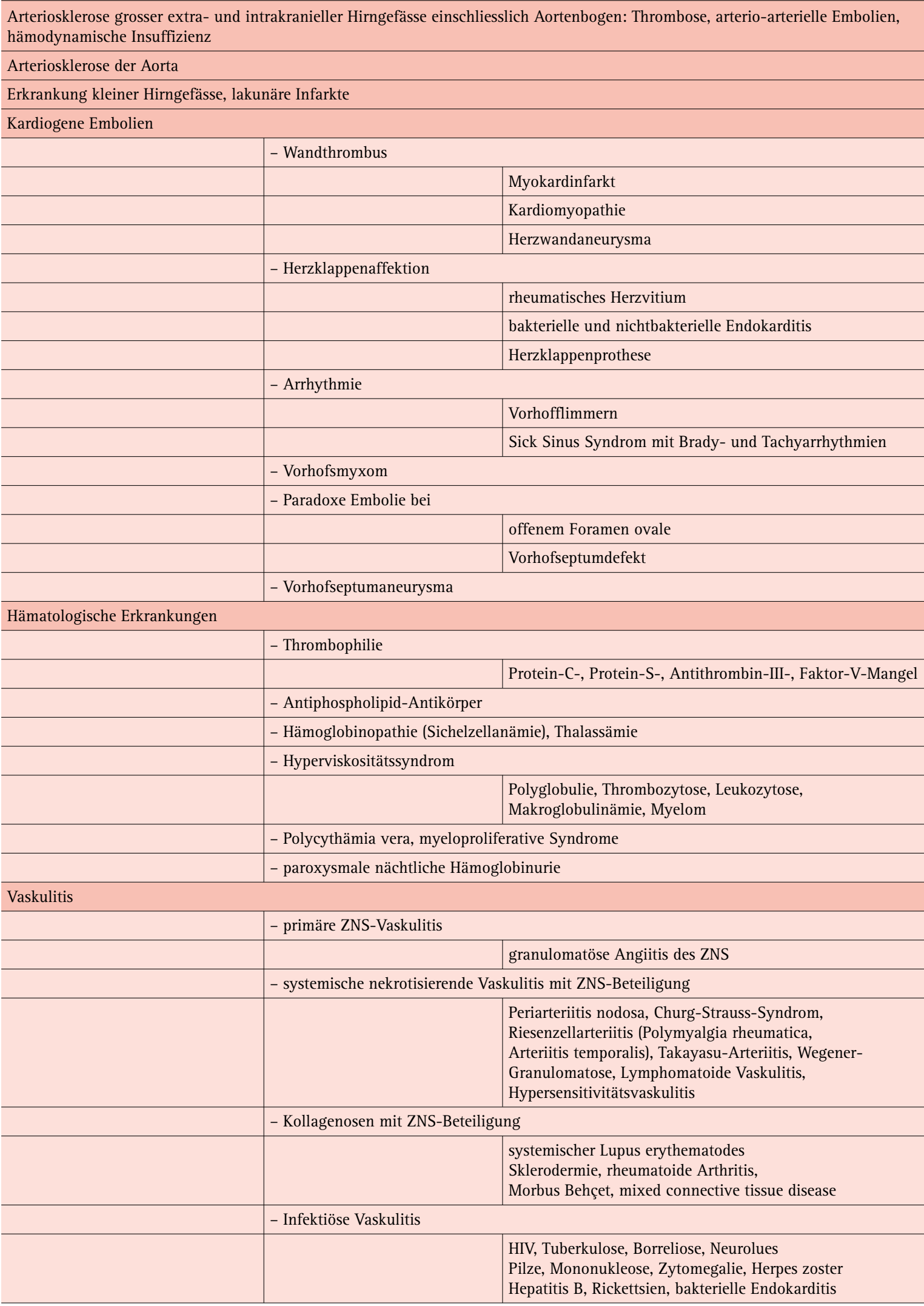




\begin{tabular}{|c|c|c|}
\hline \multicolumn{3}{|l|}{ Medikamente, Drogen } \\
\hline & \multicolumn{2}{|c|}{ - Kokain, Crack, Amphetamin, LSD, Heroin } \\
\hline & \multicolumn{2}{|c|}{ - Sympathomimetika, Ergotamine, Triptane } \\
\hline & \multicolumn{2}{|c|}{ - intravenöse Immunglobuline } \\
\hline \multicolumn{3}{|c|}{ Nichtarteriosklerotische Gefässerkrankungen } \\
\hline & \multicolumn{2}{|l|}{ - Dissektionen } \\
\hline & & $\begin{array}{l}\text { Trauma, spontan, Marfan-Syndrom, } \\
\text { fibromuskuläre Dysplasie }\end{array}$ \\
\hline & \multicolumn{2}{|c|}{ - traumatisch bedingte Thrombose oder Abriss von Hirngefässen } \\
\hline & \multicolumn{2}{|c|}{ - Vasospasmus nach Subarachnoidalblutung } \\
\hline & \multicolumn{2}{|c|}{ - Moyamoya } \\
\hline & \multicolumn{2}{|l|}{ - Dolichoektasie } \\
\hline & \multicolumn{2}{|l|}{ - Amyloidangiopathie } \\
\hline \multicolumn{3}{|l|}{ Diverse weitere Ätiologien } \\
\hline & \multicolumn{2}{|l|}{ - Homozysteinurie } \\
\hline & \multicolumn{2}{|c|}{ - Fett- und Luftembolien } \\
\hline & \multicolumn{2}{|c|}{ - Pseudovaskulitisches Syndrom mit Cholesterinembolien } \\
\hline & \multicolumn{2}{|c|}{ - Migräne } \\
\hline & \multicolumn{2}{|c|}{ - iatrogen (Angiographie, Herz- und Aortenchirurgie) } \\
\hline & \multicolumn{2}{|c|}{ - Neurofibromatose } \\
\hline & \multicolumn{2}{|l|}{ - Lungenerkrankungen } \\
\hline & & AV-Malformationen, M. Osler, Lungenvenenthrombosen \\
\hline & \multicolumn{2}{|c|}{ - distale Embolien aus Riesenaneurysmen } \\
\hline & \multicolumn{2}{|l|}{ - Tumorembolien } \\
\hline & \multicolumn{2}{|c|}{ - MELAS, CADASIL, Morbus Fabry und andere familiäre zerebrale Vaskulopathien } \\
\hline \multicolumn{3}{|l|}{ Venen- und Sinusthrombose } \\
\hline Infarkte ohne eruierbare Ätiologie & & \\
\hline
\end{tabular}

